\title{
Antecedentes históricos del término «responsabilidad» en el ámbito de la Función Pública*
}

Historical background of the term «responsibility» in the field of Public Function

Dra. Elena QUINTANA ORIVE Universidad Autónoma de Madrid elena.quintana@uam.es

Resumen: El artículo trata sobre el origen del término «responsabilidad», que surge en Inglaterra en el último cuarto del siglo XVIII para referirse a la responsabilidad política, y su recepción en países como Francia o España. Asimismo, se estudia la influencia del Derecho Romano en la formación de este sustantivo-categoría.

Abstract: The article deals with the origin of the term «responsibility», that arose in England in the last quarter of the $18^{\text {th }}$ century to refer to political responsibility, and its reception in countries such as France or Spain. Likewise, the influence of Roman Law in the formation of this noun-category is studied.

Palabras clave: Responsabilidad, funcionarios, terminología, Inglaterra, Derecho Romano, respondere, periculum, obnoxius.

Keywords: Responsibility, officials, terminology, England, Roman law, respondere, periculum, obnoxius.

Sumario:

I. La aparición del vocablo «responsabilidad» en el debate político de la Inglaterra del siglo XVIII.

* Esta contribución se enmarca dentro del Proyecto de Investigación «La construcción de un Derecho Administrativo, Medioambiental y Fiscal Romano» (DER 2017-82833-P), dirigido por los Profesores Antonio Fernández de Buján y Alfonso Agudo Ruiz. 
II. «Respondere»: precedentes romanos del término «responsabilidad».

Recibido: septiembre 2021.

Aceptado: noviembre 2021 


\title{
I. LA APARICIÓN DEL VOCABLO «RESPONSABILIDAD» EN EL DEBATE POLÍTICO DE LA INGLATERRA DEL SIGLO XVIII
}

\author{
Refiere DE ÁNGEL YAGÜEZ ${ }^{1}$ que
}

“... en un diccionario inglés de 1755, de Samuel Johnson (A Dictionary of the English Language), no aparece la palabra «responsibility», aunque sí la de «responsible», con los significados de «answerable», «accountable», probablemente como consecuencia del fenómeno del lenguaje por cuya virtud los vocablos más complejos (si se quiere más largos) son posteriores a los más sencillos. Al igual que, en español «responsabilidad» aparece más tarde que «responsable»".

El término «responsabilidad $»^{2}$ aparece por primera vez en Inglaterra, referido a la responsabilidad de los funcionarios ${ }^{3}$, en el curso del debate político parlamentario del siglo XVIII: "[...] Mr. Pitt had chosen only a Side-Place [Privy Seal], without any responsibility annexed to it [...] But a Title, and a Side-Place with a large salary, but no business or responsibility annexed to it, were baits which that gentleman had long been gaping after [...]" ("The Gentleman`s Magazine" 7/1776, XXXVI, pp. 350-351) ${ }^{4}$.

1 DE ÁNGEL YAGÜEZ, R., «Sobre las palabras "responder”, "responsable” y “responsabilidad"», en Estudios jurídicos en homenaje al profesor Luis Diez-Picazo, II, Madrid 2002, p. 1346.

${ }^{2}$ Sobre la etimología y evolución de este término, véase: LÉVY-BRUHL, L., L idée de responsabilité, Paris 1884; McKEON, R., «The Development and the Significance of the Concept of Responsibility», en Revue Internationale de Philosophie (Paris), 11.39 (1957) 3 ss.; HENRIOT, J., «Note sur la date et le sens de l'aparition du mot "responsabilité"», en Archives de Philosophie du Droit (Paris), 22 (1977) 59 ss.; VILLEY, M., «Esquisse historique sur le mot responsable», en Archives de Philosophie du Droit (Paris), 22 (1977) 45 ss.; SCHIPANI, S., «Schede sull 'origine del termine responsabilità (Contributo per una riflessione sui problemi dell'elaborazione del concetto sistematico generale designato da tale termine)», en Scritti in onore di Luigi Mengoni, I, Milano 1995, pp. 885-918 [=Contributi romanistici al sistema della responsabilità extracontrattuale, Torino 2009, pp. 1-28]; LARRAÑAGA, P., El concepto de responsabilidad, México D.F. 2000; GARCÍA DE ENTERRÍA, E., La lengua de los derechos. La formación del Derecho público europeo hasta la Revolución Francesa, Madrid 1994, pp. 142-143.

${ }^{3} \mathrm{Si}$ bien el primer testimonio escrito del sustantivo «responsabilidad» aparece en un documento francés del siglo XV de forma aislada. PROSCHWITZ, G., «Responsabilité: l' idée et le mot dans le débat politique du XVIIIe siècle», en Actes du Xe Congrès intern. de linguistique et philologie romanes, I, Strasbourg 1962, pp. 385 ss.

${ }^{4}$ PROSCHWITZ, G., «Responsabilité..., o.c., p. 389. SCHIPANI, S., «Lex Aquilia, Culpa, Responsabilità», en Illecito e pena privata in età repubblicana, Atti del Convegno Internazionale 
En este marco es utilizado también el vocablo «responsible» junto a «accountable» $\mathrm{y}$ «answerable» para referirse a la rendición de cuentas de los ministros y adoptando el significado de "estar sujeto a rendir cuentas" o de "ser moralmente imputable": "If responsibility was once removed, where could a criminal be found? Not on the throne, for the King can do no Wrong; not in Administration, for where they are not consulted, they cannot be responsible [...]" ("The Morning Herald and Daily Adviser", 18/12/1783) 5 .

En lengua inglesa, junto al mencionado vocablo «responsibility» (responsabilidad política) se encuentra el de «liability» que es utilizado en otro campo semántico: el de la responsabilidad civil extracontractual ${ }^{6}$. Esta dualidad terminológica refleja la influencia de las fuentes latinas en el derecho anglosajón que pudiendo emplear en este ámbito de la responsabilidad política otros términos como, por ejemplo, «answer» hace propio «respound» tomándolo del latín ${ }^{7}$.

Este principio político-jurídico relativo a la responsabilidad de los funcionarios así como el nuevo sustantivo-categoría «responsabilidad» serán exportados a la Francia revolucionaria de finales del siglo XVIII viniendo a caracterizar el nuevo modelo de Administración plasmado en la Declaración de los Derechos del Hombre y del Ciudadano de 26 de agosto de 1789 (art. 15: "la Société a le droit de demander compte à tout agent public de son administration") ${ }^{8}$, y el Dictionnaire de l'Academie française de 1798 consagra la palabra «responsabilité» definiéndola como: "obligation légale de répondre de ses actions, d'etre garant de quelque chose [...]. Il s'applique aux Ministres, aux hommes publics [...]”.

Por lo que hace al idioma español, el vocablo «responsable» aparece definido en el Diccionario de Autoridades de 1737 (no así la voz responsabilidad) de la siguiente forma: "El que está obligado a responder o satisfacer por algún cargo. Es voz introducida modernamente. Lat. Qui tenetur ad".

di Diritto Romano (Copanello, 4-7 giugno 1990), Napoli 1992, pp. 174, recoge que "é in questi contesti che si ritiene generalmente che sarebbe testimoniato per la prima volta responsibility (a. 1766) per la responsabilità ministeriale. «Liability» viene a coprire un altra area semantica".

${ }^{5}$ PROSCHWITZ G., «Responsalitè..., o.c., p. 391.

${ }^{6}$ En alemán, señala SCHIPANI S., «Lex Aquilia, Culpa, Responsabilità, o.c., p. 185, que "il dualismo 'Haftung'/ 'Verantwortlichkeit', così come pure il pur diverso dualismo 'Liability'/ 'Responsibility' precedentemente indicato per la lingua inglese, impongono però di sottolineare che 'responsabilità, responsabilité, ecc.' si trova, nelle lingue neolatine, in un campo semantico diversamente articolato".

${ }^{7}$ Véase SCHIPANI, S., «Lex Aquilia..., o.c., p. 172.

${ }^{8}$ En el artículo 31 del Proyecto de la Declaración de Derechos de 20-21 de julio de 1789 se establece que "les Officiers publics dans tous les genres de pouvoir sont responsables de leurs prévarications et de leur conduite". 
El término «responsabilidad» está ya presente en la Constitución de Cádiz de 1812 referido a la responsabilidad de los funcionarios y de los jueces (art. 131.25: «...Hacer efectiva la responsabilidad de los Secretarios del Despacho y demás empleados públicos») ${ }^{9}$, así como en el Proyecto de Código Civil de 1821 (arts. 7, 8 y 9) ${ }^{10}$, en el de 1836 (art. 13) y en el Proyecto de García Goyena de 1851 (art. 12) ${ }^{11}$. Entre los Códigos civiles latinoamericanos mencionamos el Código Civil de Andrés Bello (Chile, 1855) en el que aparece citado 41 veces.

\section{II. «RESPONDERE»: PRECEDENTES ROMANOS DEL TÉRMINO RESPONSABILIDAD}

Hoy en día vemos que «respondere» ${ }^{12}$ está presente en todas las lenguas modernas neolatinas («responder», en español, catalán, gallego y portugués; «répondre», en francés; «rispondere», en italiano; «raspunde», en rumano).

El término «responsabilitas» no se encuentra recogido en las fuentes jurídicas romanas ni medievales; sin embargo, sí se utiliza desde época antigua el vocablo «respondere» y otras formas derivadas del mismo como, por ejemplo, «responsum», «responsor», «responsare», «responsio»o $\left\langle\right.$ responsus ${ }^{13}$.

\footnotetext{
${ }^{9}$ De igual forma se recoge en los artículos 228, 269, 293, 294 y 372 de la Constitución de 1812.

${ }^{10}$ Art. 7: En las demás órdenes o mandatos, si se duda de su autenticidad o legitimidad por defecto de alguna solemnidad extrínseca puede suspenderse la ejecución, representando a la Autoridad de quien emanan, y bajo la responsabilidad del que debe ejecutar o cumplir, en caso de ser falso o caviloso el reparo; Art. 8: La suspensión con responsabilidad de la que habla el artículo anterior, tiene también lugar ...; Art. 9: La falta de puntualidad o de cumplimiento en la ejecución de las leyes y de los Decretos de las Cortes, de las órdenes y mandatos de las Autoridades respectivas, sujeta a los inferiores y a los subalternos a la responsabilidad y penas que establece el Código Penal.

${ }^{11}$ Art.12: El juez que rehúse fallar a pretexto de silencio, oscuridad o insuficiencia de las leyes, incurrirá en responsabilidad.

${ }^{12}$ v. Respondere, WALDE, A.- HOFMANN, J. B., Lateinisches Etymologisches Wöterbuch, 2, Heidelberg 1954, pp. 578 ss.; ERNOUT, A.- MEILLET, A., Dictionnaire étymologique de la langue latine, Paris 1967, pp. 643 ss.; CASTIGLIONI, L.- MARIOTTI, S., Vocabolario della lingua latina, Torino 1996, pp. 1267-1268. Sobre la importancia que la terminología asume en el ámbito de la experiencia jurídica romana: BIONDI, B., «La terminologia romana come prima dommatica giuridica», en Studi Arangio-Ruiz, II, Napoli 1953, pp. 73 ss.; KASER, M., «Zur juristischen Terminologie der Römer», en Studi Biondi, I, Milano 1965, pp. 97 ss.

${ }^{13}$ En época postclásica aparecen expresiones como «responsoria», «responsalis», «responsarius», «responsivus», «responsativus» o «responsorium»; y en la Edad Media surgieron nuevos vocablos como «responsaticum», «responderia», «responderius» o «responsaton». SCHIPANI, S., Contributi romanistici al sistema della responsabilità extracontrattuale, Torino 2009, p. 3; ID., «Lex Aquilia, Culpa, Responsabilità, o.c., p. 161.
} 
«Respondeo», del que derivan los términos mencionados, procede a su vez del verbo «spondeo» ${ }^{14} \mathrm{y}$ hace alusión al acto de comprometerse, de vincularse a la palabra dada a través de una promesa; con este sentido y referido a la actividad de los magistrados y del príncipe se utiliza en diversas fuentes jurídicas y literarias romanas ${ }^{15}$.

«Respondere» pasó también a la lengua común latina con el sentido general de "responder a una pregunta", y así mencionamos algunos de los contextos en los que aparece con este significado ${ }^{16}$ : para designar en la sponsio y en la stipulatio, el acto del promitente (D. 45.1.1.1-2-3: Qui praesens interrogavit, si antequam sibi responderetur [...]. Si quis ita interroget [...] responderit [...]. Si quis simpliciter interrogatus responderit [...]); para designar la respuesta a la interrogatio in iure (D. 11.1.4.1: Quod ait praetor: 'qui in iure interrogatus responderit'); para designar la respuesta de un testigo en un proceso (D. 22.5.3.1: quanta fides habenda sit testibus ... an ad ea quae interrogaveras verisimilia responderint); así como en la antigua reivindicatio (Cfr. Gai. 4,16: qui prior vindicaverat, ita alterum interrogabat ... ille respondebat). También, con este sentido, el término «respondere» pasó a aplicarse a una de las tres actividades en las cuales puede sintetizarse la labor de la jurisprudencia republicana: responder a las cuestiones o dudas planteadas por particulares, magistrados y jueces. Muchas de las creaciones de los pretores se deben al asesoramiento, a los dictámenes de los juristas ${ }^{17}$.

«Respondere» se utilizó también para hacer referencia al acto de responder a una acusación o a una citación judicial indicándose de esta forma una actividad procesa $l^{18} \mathrm{y}$, precisamente, con este sentido aparece en distintas fuentes referido a

${ }^{14}$ Festo, p. 329: Spondere Verrius putat dictum, quod 'sponte sua', id est voluntate, promittatur; deinde oblitus inferiore capite sponsum et sponsam ex Graeco dictam ait, quod ii 'spondas' interpositis rebus divinis faciant. SCHIPANI, S., Contributi romanistici al sistema della responsabilità..., o.c., p. 4; ID., «Lex Aquilia, Culpa, Responsabilità, o.c., pp. 161-162: "già gli antichi sottolineavano la connessione con la volontarietà (sponte) e il rito (spondeo), che trova in rapporto al matrimonio una risalente consolidata occasione di uso (da cui sponsalia, sponsa, sponsus ecc.) cosi come la trova in rapporto agli accordi c.d. internazionali, ed a quella sponsio che costituisce antichissima fonte di obbligazione".

${ }^{15}$ D. 1.16.6.2; D. 5.1.79.1; C. 6.38.5; Tac., Ann., 1, 77; etc... Véase, SCHIPANI, S., Contributi romanistici al sistema della responsabilità..., o.c., p. 4 .

${ }^{16}$ SCHIPANI, S., Contributi romanistici al sistema della responsabilità..., o.c., pp. 4-5.

17 FERNÁNDEZ DE BUJÁN, A., Derecho Público Romano, 23 ed., Cizur Menor 2020, p. 171; ANDRÉS SANTOS, F. J., «Roma y los juristas. El modelo romano en la jurisprudencia europea del siglo XIX», en Minerva: revista de filología clásica (Valladolid), 15 (2001) 290.

${ }^{18}$ Cic., Verr. 1,1: hanc opinionem populi Romani fuisse, C. Verrem altera actione responsurum non esse neque ad iudicium adfuturum; Asc., pro M. Scauro, 20: Iuvenibus processit in foro, deinde accepto respondendi loco dixit...; C. J. 2.12(13).2: appellationi adversariae respondere; D. 4.1.7 pr.: itaque si citatus non respondit...; Nov. 123.24.8: Si vero nondum incohata est, apud 
los funcionarios de la burocracia imperial como, por ejemplo, en CTh. 12.1.171.1 ${ }^{19}$ o C. J. 3.13.7.1 ${ }^{20}$.

El uso del vocablo «respondere» se extendió también en el ámbito del proceso a otros campos con el sentido de "cumplir" o "satisfacer" para referirse al presupuesto (deber, carga o crimen) del que deriva la posibilidad de proceder judicialmente contra alguien («respondere creditoribus», «respondere muneribus», 《respondere oneribus» ${ }^{21}$ ), tal y como se recoge en Lex Romana Wisigothorum 5.4.18: ... pro suo furto, pro crimine eius respondere vel satisfacere...

El término adoptará también en las fuentes romanas tardías y medievales el significado de "asunción de una obligación de garantía"22, utilizándose «responsalis» para referirse a aquella persona que presta una garantía $\left(\mathrm{GMIL}^{23}\right.$, 7, 150: responsalis 1: constituerunt se et fecerunt... plegios et responsales de dicto contractu [a. 1265]) y «responderius» adopta también el significado de garante (GMIL, 7, 150: responderius: pro instrumento confessionis et obligationis alicuius debiti, item mutui responderiae sibi facte pro domino nostro [a. 1330]).

Por lo que hace a nuestro objeto de estudio constatamos que el vocablo «respondere», tal y como se emplea en las fuentes romanas y referido a los funcionarios de la Administración imperial, no sólo adopta el significado de "contestar a una acusación" sino también el de "estar obligado a rendir cuentas por la gestión de un cargo público" como se refleja en las siguientes fuentes: C. J. 3.25.1 (...sub moderationibus provinciarum pro functionibus publicis respondere...); Nov. 128.24 .3 (...iubemus eum legitimo procuratore constituto conventiones pro damnis proponentibus respondere); Nov. 128.23 .17 (...post

gloriosissimum praefectum Orientis praetorium aut apud eos qui a nobis fuerint deputati iudices convenientibus eum respondere.

${ }^{19}$ Sane quoniam principalem locum et gubernacula urbium probatos administrare ipsa magnitudo deposcit, sine ordinis praeiudicio consensu curiae eligendos esse censemus, qui contemplatione actuum omnium possint respondere iudicio.

${ }^{20}$ Quapropter iubemus huiusmodi hominibus nec cuiuslibet militiae seu cinguli vel dignitatis praerogativam in hac parte suppetere, sed eos, qui statutis in quacumque militia connumerati sunt vel fuerint seu dignitatem aliquam praetendunt, sine quadam fori praescriptione his iudicibus tam in publicis quam in privatis causis oboedire compelli, ad quorum sollicitudinem professionis seu negotiationis, quam praeter militiam, ut dictum est, exercent, gubernatio videtur rescipere, ita tamen, ut ipsis nihilo minus iudicibus, sub quorum iurisdictione militia seu dignitas eorum constituta est, procul dubio respondeant.

${ }^{21}$ C. J. 9.12.2: ....Nam successio oneribus portionis suae respondet...; C. J. 1.3.48.4: ... et debita exigere et creditoribus respondere ...; C. J. 4.39.1: ... nec fiscum creditoribus hereditariis respondere ...; D. 50.1.17.9: ... muneribus civilibus respondere.

${ }^{22}$ SCHIPANI, S., «Lex Aquilia, Culpa, Responsabilità, o.c., p. 167.

${ }^{23}$ DU CANGE, CH. et al., Glossarium mediae et infimae latinitatis, Paris, ed. 1883-1887. 
depositionem cinguli quinquaginta dies in provinciis commorari et conventionis aliquas contra se moventibus respondere); ...

Junto a «respondere» se mencionan también en las fuentes jurídicas romanas dos términos: «obnoxius» y «periculum», referidos a los funcionarios y empleados públicos con el sentido de "ser responsables".

Etimológicamente, «obnoxius» $\rangle^{24}$ procede de los vocablos $o b$ y noxa o noxi $a^{25}$ y se identifica en un primer momento en el lenguaje jurídico con culpa («noxia culpa est»: Servio, In Aen, I, 41); más tarde, este significado se restringe y «noxa» se asimilará más bien a «delictum» como resulta de D. 50.16.238.3 (Gai., 6 ad leg. XII Tab.) ${ }^{26}$ : «Noxiae» apellatione omne delictum continetur $^{27}$. Añadimos que de esta palabra-raíz derivan a su vez los términos «noxius» con el sentido de "culpable o responsable en virtud de un delictum" e «innoxius» como "ausente de responsabilidad".

La locución «obnoxius» toma en las fuentes jurídicas y literarias latinas el sentido de "sujeto a pena", "punible", "culpable", "obligado", "responsable"28. En este sentido, MURGA GENER señalaba que la expresión «obnoxius» es clásica y que "implica una idea de sometimiento cuya primera acepción debió ser la derivada de una responsabilidad penal o criminal, obnoxius poenae (D. 29.5.8 pr., Paul., 46 ad ed.; C. J. 2.55(56).2, Caro, Carino y Numeriano, a. 283), obnoxius lege Aquilia (D. 11.3.14.7, Paul., 19 ad ed.; C. J. 3.35.3, Gordiano, a. 241).

24 «Obnoxius» es un vocablo utilizado frecuentemente en el lenguaje de la Cancillería imperial: aparece citado 37 veces en el Código Teodosiano, 36 en el Código de Justiniano y en 8 ocasiones en el Digesto.

${ }^{25}$ BIONDI, B., «Noxa», en N.N.D.I., 11, Torino 1965, p. 449; BRACHET, J.-P., «Obligare et obnoxius: observations morphologiques et sémantiques», en Glotta: Zeitschrift für Griechische und Lateinische Sprache (Göttingen), 81 (2005) 32, nos dice que "noxa et son doublet noxia sont de vieux termes juridiques, connus depuis les XII Tables (Tabula XII.1: si servo furtum faxit noxiamve noxit)".

${ }^{26}$ Nos refiere BISCARDI, A., «Noxae deditio sui», en Illecito e pena privata in età repubblicana. Atti del convegno internazionale di diritto romano (Copanello 4-7, giugno 1990), Napoli 1992, p. 99, que: "noxa e noxia sono i termini adoperati in antico per designare quello che assai più tardi sarà, nel diritto preclassico e classico, il delictum o maleficium, sancito da azione civile".

${ }^{27}$ La palabra «noxia» abarca todos los delitos.

${ }^{28}$ BRACHET, J.-P., «Obligare et obnoxius..., o.c., p. 32: "Obnoxius, de son côté, est un terme juridique archaïque dont les emplois se sont élargis". El término «obnoxius» se encuentra recogido ya en Tito Livio referido a la abolición del nexum por la lex Poetelia Papiria en torno al 326 a. C. (Ab urbe condita, 8, 28, 8: victum eo die... ingens vinculum fidei: iussique consules ferre ad populum, ne quis, nisi qui noxam meruisset, donec poenam lueret, in compedibus aut in nervo teneretur; pecuniae creditae bona debitoris, non corpus obnoxium esset). Cfr. Cicerón, de re publ. 2.34.59; Varrón, de lingua latina, 7.105. Véase, BISCARDI, A., «Noxae deditio sui», en Illecito e pena privata in età repubblicana, o.c., p. 100. 
Más tarde, esta idea originaria de culpabilidad se extendió también a otros supuestos de obligaciones de naturaleza pública. En este sentido se usa obnoxius, por ejemplo, para designar a los deudores del fisco, C. J. 10.2.4, Valentiniano, Valente y Graciano, a. 369"29. Y de esta manera se recoge en diversas fuentes referido a los funcionarios de la Administración imperial romana como CTh. 2.1.3 (Non solum in agentes in rebus, sed in alios etiam cuiuslibet homines condicionis competentes vigoris aculei exserantur, si modo fuerit declaratum delicto eos obnoxios detineri... Quae obnoxios claruerit invasisse, in duplum restituere compellantur); C. J. 10.48(47).2 (... Si quis autem usurpatoria temeritate amplius aliquid fuerit conatus exigere, obnoxius quadrupli repetitione teneatur); o C. J. 9.28.1 (Iudices, qui tempore administrationis publicas pecunias subtraxerunt, lege Iulia peculatus obnoxii sunt et capitali animadversioni eos subdi iubemus...).

Por lo que hace al término «periculum» vemos que el mismo ha sido estudiado por la doctrina romanística principalmente en el ámbito del incumplimiento de las obligaciones, adoptando el significado de riesgo (periculum emptoris) y referido a situaciones de responsabilidad objetiva. Sin embargo, $\mathrm{VOCI}^{30}$, en un estudio realizado sobre la responsabilidad de los cotutores y de los administradores de las ciudades (magistrados y curatores rei publicae), consideró que el término «periculum» describe frecuentemente, en este ámbito, una situación de responsabilidad subjetiva: "Periculum tutoris è usato di frecuentemente per denotare ciò di cui un tutore risponde: la traduzione più adatta sembra, appunto, quella di responsabilità. Mutuum periculum è un modo di essere responsabile: la situazione in cui uno risponde per l altro". También

${ }^{29}$ MURGA GENER, J. L., «La "obnoxietas” una esclavitud legal», en Règle et pratique $d u$ droit dans les réalités juridiques de l'antiquité (Atti della $51^{\circ}$ Sessione della SIHDA, CrotoneMessina, 16-20 settembre 1997), Catanzaro 1999, p. 444 nt. 13. El término «obnoxius» se utiliza también en las fuentes de época postclásica para referirse a la vinculación que los corporati (navicularii, monetarii, murileguli...) tenían con su corporación. Véase también el resumen de la comunicación presentada por A.J.B. SIRKS en el Congreso SIHDA de 1991 y recogida en RIDA (Bruxelles, 1992): «"Obnoxietas". Responsibility for public functions», concretamente en p. 441 nos dice: "Le terme «obnoxietas» $n$ ' est pas atteste avant le milieu du cinquiéme siècle après J.C. Il indique la responsabilité du citoyen pour l'execution des charges, devoirs et fonctions publiques (munera et honores) dans l'empire romain".

${ }^{30}$ VOCI, P., «La responsabilità dei contutori e degli amministratori cittadini», en IURA (Napoli), 21 (1970) 143 y nt. 221; ID., «La responsabilità dei contutori e degli amministratori cittadini - Contributi allo studio della mutua garanzia», en Studi in memoria di G. Donatuti, vol. III, Milano 1973, pp. 1305 ss. En este sentido, MacCORMACK, G., «Further on "periculum"», en B.I.D.R (Milano), 21 (1979) 12, ha señalado que: "Periculum is prominent in areas of law other than contract. Many decisions relating to dos, tutela and cura are framed in the language of periculum. Discussions of the liability of magistrates and other officials frequently make use of the same term and it is also found in decisions on the operation and assignment of particular actions". Sobre el término periculum en otros ámbitos, véase recientemente, SANTINI, P., «Periculum/ pariculum, una querelle filologico-giuridica tra tradizione testuale e Begriffsgeschichte», en Teoria e storia del diritto privato (www. Teoriaestoriadeldirittoprivato.com), 10 (2017) 1 ss. 
MacCORMARK ${ }^{31}$ ha señalado que: "The use of periculum in the context of liability incurred by magistrates, officials and clergy has some affinity with its use in the context of tutela".

Así, vemos que la palabra «periculum» aparece utilizada en numerosas ocasiones referida a la responsabilidad de los funcionarios en general ${ }^{32} \mathrm{y}$, en especial, a la responsabilidad de los magistrados y de los curatores rei publicae frente a la civitas, como se recoge, entre otros fragmentos, en: CTh. 12.5.1: Ii magistratus, qui sufficiendis duumviris in futurum anni officium nominationes impertiunt, periculi sui contemplatione provideant...; C. J. 11.36(35).2: Quotiens duobus non separatim, sed pro indiviso munus iniungitur, et ita, ut unusquisque eorum periculo soliditatis videatur obstrictus...; D. 27. 8.1 .9 (Ulp., 36 ed.): Si inter magistratus hoc convenerit, ut alterius tantum periculo tutores darentur, conventiones pupillo non praeiudicare divus Hadrianus rescripsit ...; D. 50.1.11 pr (Pap., 2 quaest.): Imperator Titus Antoninus Lentulo Vero rescripsit magistratuum officium individuum ac periculum esse commune. Quod sic intellegi oportet, ut ita demum collegae periculum adscribatur...; D. 50.1 .13 (Papir., 2 de const.): ...talis ordo dabitur, ut in primis qui rei publicae negotia gessit et qui pro eo caverunt in solidum conveniantur, mox peractis omnibus periculum adgnoscat qui non idoneum nominabit (...) Nec iuste qui nominabit universi periculum recusabit, cum scire deberet eum qui nominaretur individuum officium et commune periculum suscepturum; D. 50.8.4(3) (Pap., 1 resp.): Curatores communis officii divisa pecunia, quam omnibus in solidum publice dari placuit, periculo vice mutua non liberantur...

«Periculum» se recoge en numerosas constituciones para referirse tanto a la responsabilidad por actos u omisiones del funcionario en el desempeño de su cargo $^{33}$, como a la responsabilidad del funcionario por los actos u omisiones realizados por un sustituto suyo, el cual desempeña sus funciones "periculo suarum facultatum" 34 , y también a la responsabilidad por los actos de subordinados $^{35}$, colegas $^{36}$ o de personas por él nombradas ${ }^{37}$.

${ }^{31}$ MacCORMACK, G., "Further on «periculum», o.c., p. 25; p. 28: "Generally therefore it seems reasonable to conclude that magistrates and officials in many cases were made strictly liable and that such liability was described in terms of periculum".

${ }^{32}$ Como, por ejemplo, C. J. 12.22(23).1: Curiosi et stationarii, vel quicumque funguntur hoc munere, crimina iudicibus nuntianda meminerint et sibi necessitatem probationis incumbere, non citra periculum sui, si insontibus eos calumnias nexuisse constiterit; o C. J. 12.59(60).10: ...sed periculo primatum uniuscuiusque officii ipsas authenticas sacras...

${ }^{33}$ Véase, por ejemplo: C. J. 1.9.17: Iudaeorum primates..., periculo suo anniversarium canonem de synagogis omnibus palatinis compellentibus exigant ad eam formam...; C. J. 4.46 .2 pr.: ...quibus periculum exactionis tributorum imminet; C. J. 11.71(70).1 pr.: ...ut periculo collocantium officiorumque tradantur.

${ }^{34}$ C. J. 1.3.21: ...per substitutos periculo suarum facultatum curiae muneribus satisfacere non vetentur; C. J. 10.32(31).60.1: ...qui honorario titulo illustrem dignitatem consecuti sunt, 
Ahora bien, añade también MacCORMACK ${ }^{38}$ que algunos de los textos acerca de la responsabilidad de magistrados y funcionarios utilizan «periculum» con el sentido de sujeto a pena (liability to a penalty): periculum sententiae, periculo carceris, periculum criminis, periculo calumniae, periculo infamiae y otras expresiones similares (CTh. 9.38.7; C. J. 7.55.2; C. J. 9.6.4.1; C. J. 9.1.15; C. J. 4.34.10).

per substitutos periculo suarum facultatum curiae muneribus satisfacere non vetentur; C. J. 12.20.5.2 (21.1): ...electione sua suarumque periculo facultatum praefatum munus eos implere praecipimus.

${ }^{35}$ C. J. 11.59(58).3; C. J. 10.1.9; C. J. 10.23.3.3: ...periculo rectorum provinciarum ...; D. 50.8(9).11 pr. (Papir., 2 const.): ...eius vero, quae a redemptoribus operum exigi non potest, sortis dumtaxat periculum ad curatores pertinere.

${ }^{36}$ C. J. 10.2.3 pr.: qui exactores fuerant nominati, confusae exactionis mutuum periculum constitutum ...; D. 50.1 .11 pr. (Pap., 2 quaest.): ... magistratuum officium individuum ac periculum esse commune ... collegae periculum adscribatur.

${ }^{37}$ C. J. 10.1.9; C. J. 1.32 .1 pr.: Palatinis haec cura debet esse praecipua, ut, periculo proprio notariis destinatis, super neglegentia iudicum, si ita res exegerit, conquerantur, ne eorum sit impunita desidia.

${ }^{38}$ MacCORMACK, G., «Further on periculum..., o.c., p. 29. 
